\title{
Recent COMPASS results on Transverse Spin and Momentum dependent distributions and fragmentation functions
}

\author{
Andrea Bressan*广 \\ University of Trieste and Trieste Division of INFN, Trieste, Italy \\ E-mail: Andrea.Bressan@cern.ch
}

COMPASS is a fixed target experiment in operation at CERN since 2002 with a wide physics programme. By using either muon or hadron beams, both the study of the spin structure of the nucleon and hadron spectroscopy studies focusing on the search of exotic states are performed. An important part of this programme is the study of transverse spin and momentum dependent parton distribution and fragmentation functions (TMD PDFs and FFs). First measurements of HERMES and COMPASS showed sizeable Collins and Sivers asymmetries, allowing first extractions of spin-dependent TMD PDFs and FFs. Now this field of research is entering the phase of precision measurements and is a central topic of many present and future experiments.

Recent COMPASS results on Collins and Sivers asymmetries and on multidimensional analysis of SSA will be reviewed together with the perspective for future measurements.

XXIV International Workshop on Deep-Inelastic Scattering and Related Subjects

11-15 April, 2016

DESY Hamburg, Germany

*Speaker.

${ }^{\dagger}$ On Behalf of the COMPASS Collaboration. 


\section{Introduction}

The study of transverse momentum and transverse spin dependent effects in COMPASS started in 2002, using the transversely polarised deuterium target and collecting only a marginal $20 \%$ fraction of the total data sample obtained with the polarised target. Since then the interest in TMD PDFs and FFs has grown significantly both theoretically and experimentally. Evidence of the existence of interactions coming from spin-orbit correlations inside the nucleon and in the fragmentation process has been found experimentally by HERMES [1,2], COMPASS [3,4], BELLE and BaBar [5,6]. On the theory side the description of the nucleon at leading twist $[7,8]$ has been done and QCD factorisation theorems $[9,10]$ proven leading to universality (or pseudo-universality) of the TMDs (see f.i. [11-13] for recent reviews).

Spin couples naturally to the intrinsic transverse momentum $k_{\perp}$ of quarks; at the leading twist, this results in eight nucleon TMD PDFs describing different spin-transverse momentum correlations, such as the correlation of the quark transverse momentum $k_{\perp}$ and the nucleon transverse spin (the most famous Sivers function $f_{1 T}^{\perp, q}$ ) or the quark transverse spin with its transverse momentum, inside an unpolarised nucleon (the Boer-Mulders function $h_{1}^{\perp, q}$ ). On the fragmentation function side, the Collins $H_{1}^{\perp}$ FFs plays a special role. $H_{1}^{\perp}$ in fact may acts as a polarimeter being sensitive to the correlation between the transverse momentum gained during fragmentation and the transverse polarisation of the fragmenting quark [14] and allows to access the chirally-odd PDFs.

In the SIDIS $\ell(l)+N(P) \rightarrow \ell\left(l^{\prime}\right)+h\left(p_{h}\right)+X$ cross-section, the convolution of TMD PDFs and FFs are describing the strength of modulations as a function of the azimuthal angle of hadrons $\phi_{h}$ and quark transverse spin $\phi_{S}$ [8]. To further separate the individual contribution of the PDFs and FFs one needs to combine the analysis of SIDIS experiments with those of $e^{+} e^{-}$collider experiments where the modulations depend on convolutions of FFs only or Drell-Yan experiments where the convolution is between two PDFs. Doing global fits of SIDIS, Drell-Yan and $e^{+} e^{-}$ experiments further requires to evolve the TMD PDFs and FFs to the measured experimental points, with the evolution kernel of TMDs still being investigated with different approaches. There are five Single-Spin Asymmetries (SSA), which depend only on the transverse target spin $S_{T}$ and three Double-Spin Asymmetries (DSA), both $S_{T}$ and $P_{B}$ (beam polarisation) dependent. In the QCD parton model approach four of the eight TSAs have leading twist interpretation. The remaining four asymmetries are higher-twist effects, though they can be interpreted as Cahn kinematic corrections to twist-two spin effects on the transversely polarised nucleon. In the following only Collins, twohadron and Sivers asymmetries will be shortly discussed.

\section{The Collins asymmetry}

The main source of information on the transversity PDFs is at present the Collins asymmetry, which couples $h_{1}$ to the Collins fragmentation function $H_{1}^{\perp}$ :

$$
A_{U T}^{\sin \left(\phi_{h}+\phi_{S}\right)}(x) \simeq \frac{\sum_{q} e_{q}^{2} h_{1}^{q}(x) \otimes H_{1}^{\perp, q \rightarrow h}}{\sum_{q} e_{q}^{2} f_{1}^{q}(x) \otimes D_{1}^{q \rightarrow h}}
$$

with $\otimes$ indicating convolution integrals between the quark transverse momentum and the transverse momentum acquired by the hadron in the fragmentation process with respect to the quark. In the 
first analysis the asymmetries as a function of one kinematic variable were obtained by integrating over the other kinematic variables. The Collins asymmetry has been measured by COMPASS on deuterons $\left({ }^{6} \mathrm{LiD}\right)[15,16]$ and on protons $\left(\mathrm{NH}_{3}\right)[4,17]$ for unidentified hadrons (dominated by pions), $\pi$ and $K$. Keeping in mind the COMPASS convention for the Collins angle, the Collins asymmetries measured for charged $\pi$ on protons show a clear negative signal for $\pi^{+}$and a positive signal for $\pi^{-}$as a function of $x, z$ and $p_{\mathrm{T}}$. The asymmetries measured by COMPASS and HERMES have the same amplitude once the HERMES data are corrected for the depolarisation factor, giving important information on the kinematic dependencies of the transversity PDF and Collins FF. The trend is confirmed for $K^{+}$, while $K^{-}$and $K^{0}$ signals are smaller (or absent). The null COMPASS results on deuteron allows to constrain $h_{1}^{d}$. A global analysis of COMPASS, HERMES and Belle data allowed first extractions of transversity PDFs and Collins FFs $[18,19]$, showing that transversity function is sizeable and different from zero, with opposite sign and similar amplitude for the $u$ and $d$ quark, while at the same time favoured and unfavoured Collins FF are also large, with similar amplitude but opposite sign. The next step of the COMPASS analysis is the extrac-

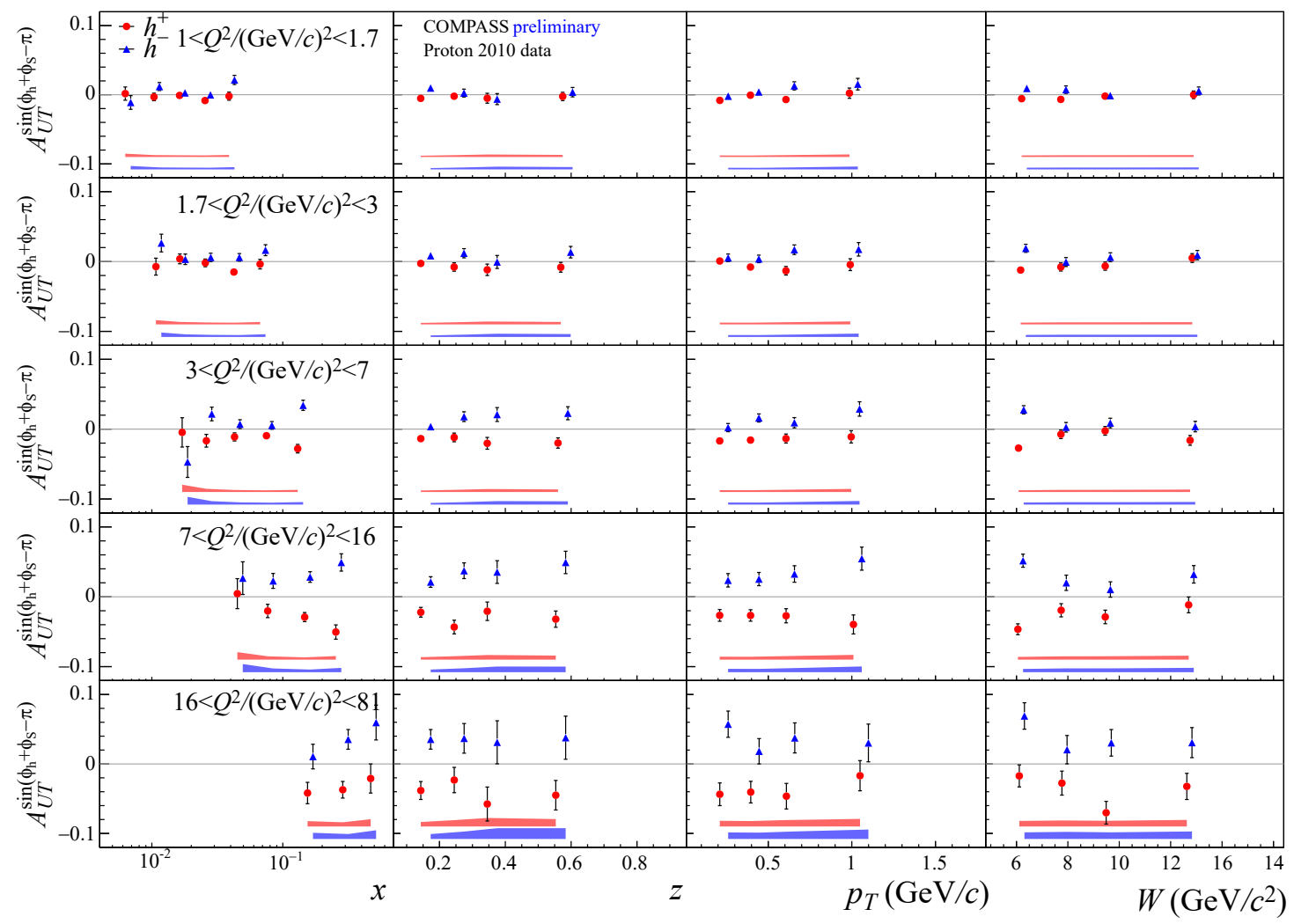

Figure 1: Collins asymmetries for positive (red) and negative (black) hadrons for different $Q^{2}$ bins, as a function of $x$ (left), $z$ (middle-left) and $p_{\mathrm{T}}$ (middle-right) and $W$ (right). The results shown are for $z>0.1$ and $p_{\mathrm{T}}>0.1 \mathrm{GeV} / c$.

tion of the asymmetries in multi-dimensional bins of the kinematic variables, as shown in Fig. 1. In this example, the Collins asymmetry is shown as a function of $x, z, p_{\mathrm{T}}$ and $W$ for different $Q^{2}$ ranges, thus allowing first direct check of TMD evolution framework, even if in a limited kinematic range. The precision of the measurement is remarkable on most of the available phase-space; the 
asymmetries show mirror symmetry between positive and negative hadrons, even when the going in multidimensional bins and interesting trends are visible. The errors increase considerably going to higher $Q^{2}$ or $x$.

\section{Two-hadron asymmetry and interplay with the Collins asymmetry}

The transverse spin asymmetry in the distribution of the azimuthal plane of oppositely charged hadron pairs in the current jet of DIS have been measured by COMPASS both with the deuteron and the proton targets. The analysis of the proton data has shown that there is a striking similarity among the Collins asymmetry for positive and for negative hadrons [20] and the di-hadron asymmetry as functions of $x$, as clearly shown in Fig. 2 (left). First, the Collins asymmetries for positive and for negative hadrons have the same magnitude but opposite sign, as can be explained assuming $h_{1}^{u} \simeq-h_{1}^{d}$ and opposite sign for favoured and unfavoured Collins fragmentation functions [19]. Secondly, the di-hadron asymmetry is slightly larger in magnitude, but very close to the values of the absolute Collins asymmetry for negative hadrons. Thus the analysing powers of the single and di-hadron channels are almost the same. The correlations between the azimuthal angle of the two
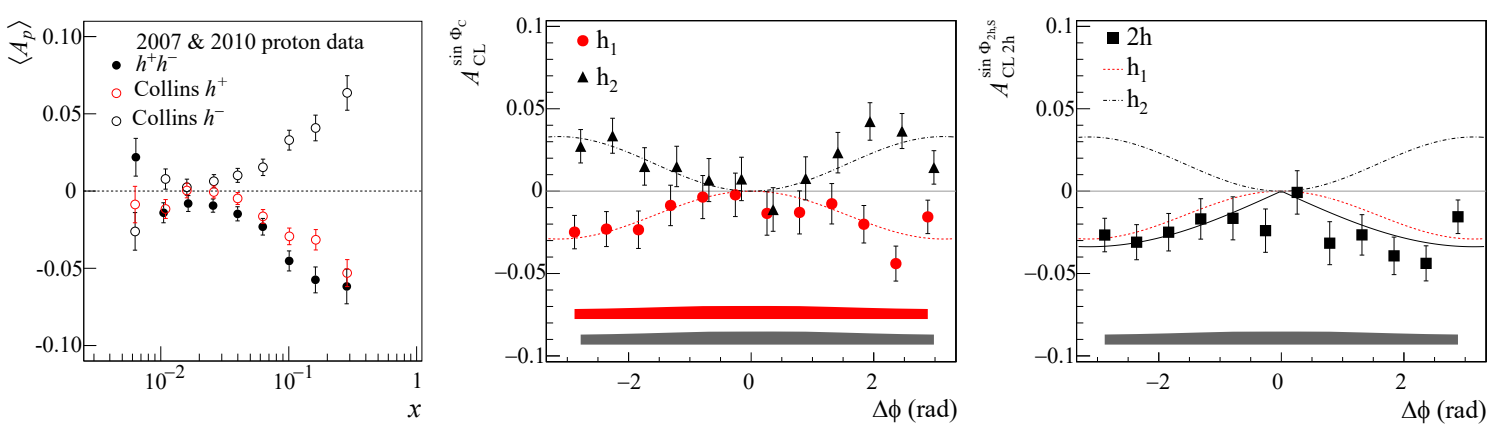

Figure 2: (left) Comparison of the asymmetry vs. $x$ obtained in the analysis of di-hadron production to the corresponding Collins asymmetry for the combined 2007 and 2010 data. (centre) The $A_{C L 1}^{\sin \Phi_{C 1}}$ (red circles) and the $A_{C L 2}^{\sin \Phi_{C 2}}$ (black triangles) vs $\Delta \phi$. Superimposed are the fitting functions described in the text. (right) $A_{C L 2 h}^{\sin \Phi_{2 h, S}}$ vs $\Delta \phi$ and the corresponding fit (black full curve). The dashed red and dot-dashed black curves are the fits to $A_{C L 1}^{\sin \Phi_{C 1}}$ and $A_{C L 2}^{\sin \Phi_{C 2}}$ from the (centre).

hadrons $\phi_{1}$ and $\phi_{2}$ in the SIDIS structure functions describing target transverse polarisation dependence [21] had been investigated by measuring the Collins 'like' (CL, i.e. Collins asymmetries in the two hadron subsample ${ }^{1}$ ) asymmetries for the first, the second and the two hadrons as functions of $\Delta \phi=\phi_{1}-\phi_{2}$ [22], where subscript 1 (2) stands for positive (negative) hadron. The results are shown in Fig. 2 (centre and right). The two single hadron asymmetries are even functions of $\Delta \phi$, compatible with zero for $\Delta \phi=0$, and increase in magnitude as $\Delta \phi$ increases. Very much as in Fig. 2 (left) the mirror symmetry between positive and negative hadrons is a striking feature for $h_{1}$ and $h_{2}$ also when the asymmetry is shown with respect to $\Delta \phi$. The last step of the COMPASS investigation [22] has been the formal derivation of a connection between the $\mathrm{CL}$ and the di-hadron asymmetries and the comparison with the experimental data. The azimuthal angle $\phi_{2 h}$ of the vector

\footnotetext{
${ }^{1}$ It has been verified the Collins and Collins like asymmetries are compatible.
} 
$\vec{R}_{N}=\hat{p}_{T 1}-\hat{p}_{T 2}$ is evaluated for each pair, with the hat indicating unit vectors. Introducing the angle $\Phi_{2 h, S}=\phi_{2 h}+\phi_{S}-\pi$, it was shown [20] that the di-hadron asymmetry measured from the amplitude of $\sin \Phi_{2 h, S}$ is essentially identical to the standard di-hadron asymmetry. Using the experimental result $\sigma_{C 2} / \sigma_{U}=-\sigma_{C 2} / \sigma_{U}=\operatorname{Const}(\Delta \phi)$ while deriving the relation between the single-two hadron cross-section it has been shown that the two cross-section implied a sine modulation in $\Phi_{C 1}$ and $\Phi_{2 h, S}$ respectively with amplitudes

$$
A_{C L 1}^{\sin \Phi_{C 1}}=\frac{1}{D_{N N}} \frac{\sigma_{C 1}}{\sigma_{U}} \cdot(1-\cos \Delta \phi) \quad A_{C L 2 h}^{\sin \Phi_{2 h, S}}=\frac{1}{D_{N N}} \frac{\sigma_{C 1}}{\sigma_{U}} \cdot \sqrt{2(1-\cos \Delta \phi)} .
$$

In Figure 2 (right) the $A_{C L 2 h}^{\sin \Phi_{2 h, S}}$ asymmetry is shown together with the curve $c \sqrt{2(1-\cos \Delta \phi)}$. The dashed red and dot-dashed black curves are the fitted curves $a(1-\cos \Delta \phi)$ of Fig. 2 (centre). The ratio of the integrals of the di-hadron amplitudes over the CL amplitude for positive hadron in two hadron sample agrees with the expected value $4 / \pi$ and with the original COMPASS observation that the di-hadron asymmetry is somewhat larger than the Collins asymmetry for positive hadrons.

\section{The Sivers asymmetry}

The difference between the probability to find an unpolarised quark with transverse momentum $\vec{k}_{\perp}$ and the probability to find it or with transverse momentum $-\vec{k}_{\perp}$ inside a transversely polarised nucleon is proportional to the Sivers function $f_{1 T}^{\perp}$ and modulated by the time-reversal odd correlations $\left(\hat{P} \times \vec{k}_{\perp}\right) \cdot \vec{S}$. If not null, such azimuthal asymmetry will therefore show that unpolarised quarks in a transversely polarised nucleon have a preferential motion direction. It was shown that in order not to vanish this TMD PDF requires final state interactions in SIDIS, i.e. only gluon exchanges between the struck quark and the target remnant can generate a non-zero Sivers asymmetry. Since time reversal changes a future-pointing Wilson line into a past-pointing one, T-invariance, rather than constraining $f_{1 T}^{\perp}$ to zero, gives a relation between processes that probe Wilson lines pointing in opposite time directions. In particular, since within QCD factorization framework in SIDIS the Sivers asymmetry arises from the interaction between the nucleon spectator and the outgoing quark, whereas in Drell-Yan production it arises from the interaction between the incoming quark from transitively polarised hadron and the spectator of another initial hadron, one gets a process dependent prediction for the Sivers function:

$$
\left(f_{1 T}^{\perp}\right)_{\mathrm{SIDIS}}=-\left(f_{1 T}^{\perp}\right)_{\mathrm{DY}}
$$

This relation is a direct consequence of the gauge structure of parton distribution functions, and its experimental check, presently carried on by COMPASS with the first polarised Drell-Yan data collected last year, is extremely important.

The main source of information on the quark Sivers PDFs is the measurement of the Sivers asymmetry in SIDIS, which couples $f_{1 T}^{\perp}$ to the unpolarised fragmentation function $D_{1}$ :

$$
A_{U T}^{\sin \left(\phi_{h}-\phi_{S}\right)}(x) \simeq \frac{\sum_{q} e_{q}^{2} f_{1 T}^{\perp, q}(x) \otimes D_{1}^{q \rightarrow h}}{\sum_{q} e_{q}^{2} f_{1}^{q}(x) \otimes D_{1}^{q \rightarrow h}}
$$


The Sivers asymmetry has been measured by COMPASS on deuterons $\left({ }^{6} \mathrm{LiD}\right)[15,16]$ and on protons $\left(\mathrm{NH}_{3}\right)[17,23]$ for unidentified hadrons (dominated by pions), $\pi$ and $K$ ). For both COMPASS [23] and HERMES [1] the asymmetries on polarised protons for negative pions and kaons, as well as for neutral kaons are compatible with zero, while for positive pions and kaons there is a clear evidence for a positive signal. For COMPASS the signal extends over the full measured $x$ region and increases with $z$. As for HERMES, the $K^{+}$signal is larger than the $\pi^{+}$one, which indicates a possibly not negligible role of sea quarks. Unlike the case of the Collins asymmetry, the Sivers asymmetry measured by COMPASS at large $x$ for positive pions and kaons is smaller than the one from HERMES. Several fits, which also include the recently revisited $Q^{2}$ evolution framework, were performed using HERMES asymmetries, COMPASS asymmetries on deuteron and for unidentified hadrons on proton, and JLab Hall A asymmetries on ${ }^{3} \mathrm{He}$. Some of these fits [24-26], which employ $Q^{2}$ TMD evolutions, are able to reproduce well the results of the three experiments.

Also in this case the next step of the analysis is coming from the multi-dimensional asymmetries. Figs. 3 shows a picture of the $Q^{2}$ evolution of Sivers asymmetry at COMPASS kinematics. At fixed $x$ bins (rows) the measured asymmetries as a function of $Q^{2}$ are shown for different $z$ and $p_{T}$ ranges (columns). Different non trivial trends of the data are visible. The possibility to better fix the $Q^{2}$ evolution from this data is encouraging. The relative large statistics collected by COMPASS with a proton target allows to study, within a single $x$ bin, the behaviour of the asymmetries as a function of $Q^{2}$. This is particularly important also in view of the planned Drell-Yan measurement that will check the expected change of sign of the Sivers asymmetry function, selecting events above $J / \psi$ resonance, i.e. $Q^{2}>16(\mathrm{GeV} / c)^{2}$. Non-zero effects were detected for asymmetries in all ranges. A clear signal is visible for the Sivers asymmetry with positive hadrons in all ranges and some hints of possible non-zero effect can be noticed for negative hadrons at relatively large $x$ and $Q^{2}$.

The quark Sivers function has an exact gluonic counterpart, $f_{1}^{g \perp}$, which represents the distribution of unpolarised gluons in a transversely polarised hadron. COMPASS has recently measured the Sivers asymmetry for gluons from transversely polarised ${ }^{6} \mathrm{LiD}$ [27] and $\mathrm{NH}_{3}$ [28] data. The gluon Sivers asymmetry was extracted using a technique similar to the one adopted for the gluon helicity $\Delta g$ analysis [29,30], namely measuring the azimuthal asymmetry of total momentum of a pair of hadrons produced with high transverse momentum. This selection enhancing
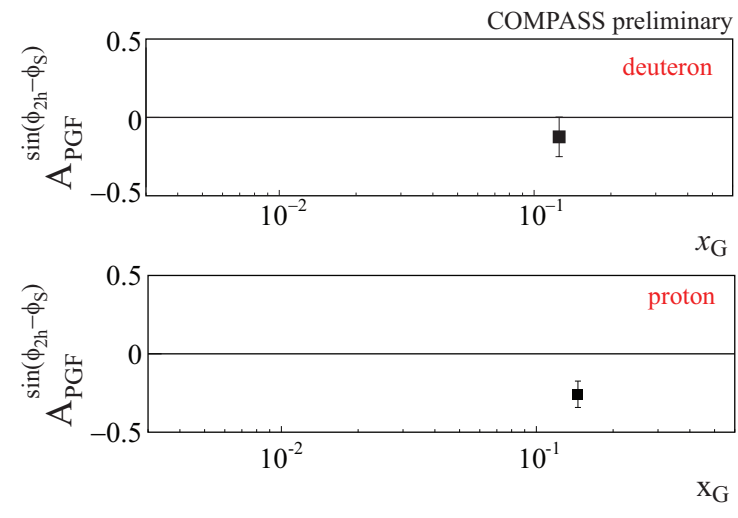

Figure 4: COMPASS Sivers asymmetry for gluons on deuterons (top) and on protons (bottom). the contribution of photon-gluon hard scattering. As originally expected from phenomenological studies of existing data [31,32] the asymmetry on the deuteron was found small (Fig. 4) and compatible with zero, within the present experimental precision. But the gluon Sivers asymmetry measured in the higher statistics proton data appears to be large and negative - a quite interesting and unexpected result. 


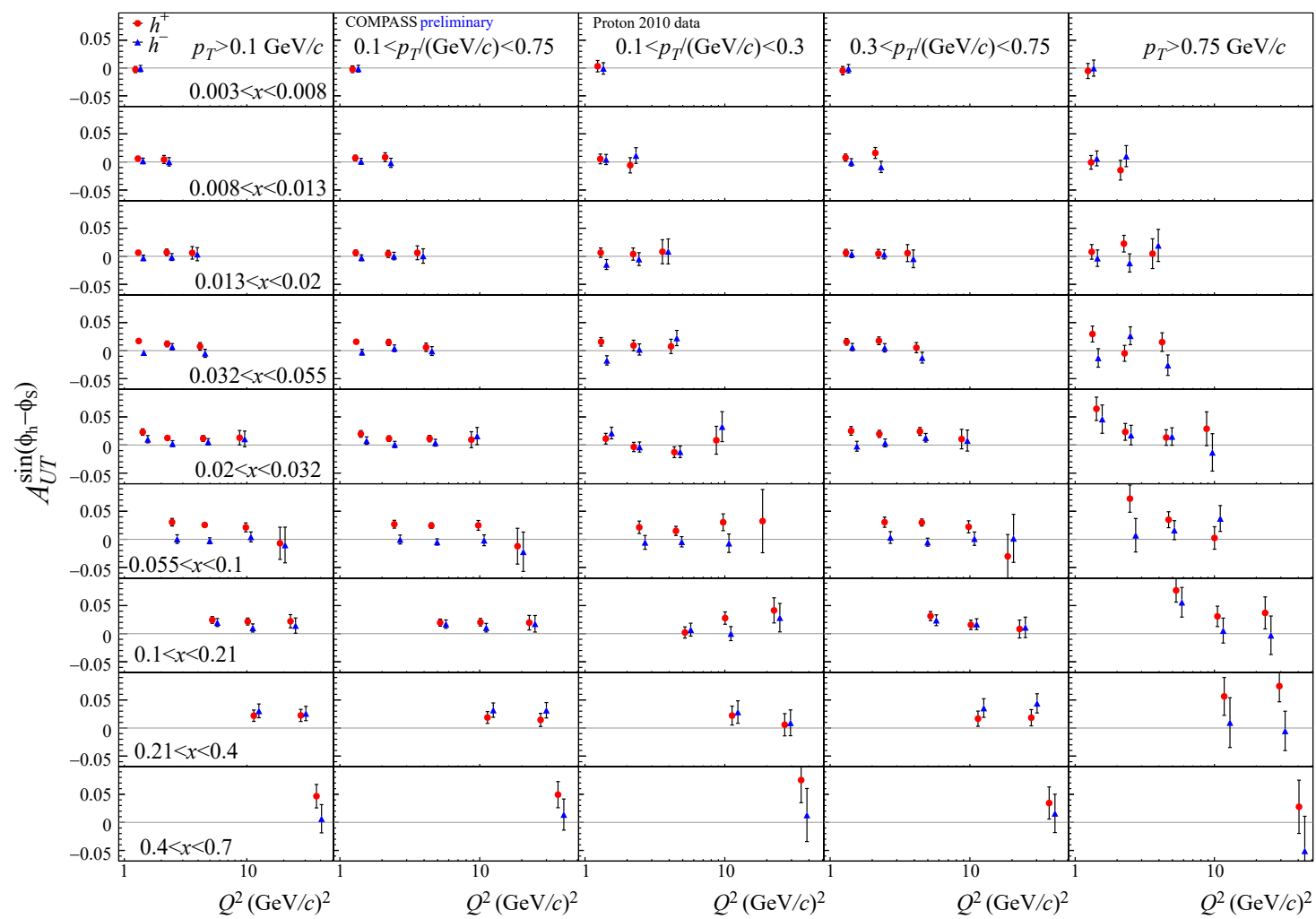

Figure 3: COMPASS measurements of the Sivers asymmetries for positive (red) and negative (black) hadrons for different $x$ bins (rows), as a function of $Q^{2}$ for $z>0.1$. The five columns refer to different $p_{T}$ ranges: (left) $p_{T}>0.1 \mathrm{GeV} / c$, (left-centre) $0.1 \mathrm{GeV} / c<p_{T}<0.75 \mathrm{GeV} / c$, (centre) $0.1 \mathrm{GeV} / c<p_{T}<0.3$ $\mathrm{GeV} / c$, (centre-right) $0.3 \mathrm{GeV} / c<p_{T}<0.75 \mathrm{GeV} / c$ and (right) $p_{T}>0.75 \mathrm{GeV} / c$.

\section{Conclusion}

COMPASS contribution to the study of the transverse spin and transverse momentum structure of hadrons has been and will be important. TMDs are now a central field of research of many laboratories around the world. In the last years, relevant experimental findings, like that there is a correlation between the spin of transversely polarised quarks and the $p_{\perp}$ of the hadrons created in the quark hadronisation process or that there is also a non-zero correlation between the spin of a transversely polarised nucleon and the intrinsic transverse momentum of the quarks, have been achieved.

Nevertheless many things remain to be done; the available SIDIS data cover only a limited phase space, and many more data are needed to obtain the $p_{\mathrm{T}}$ and $Q^{2}$ dependence of the asymmetries in the different $x$ and $z$ bins, that are needed to allow model independent extraction of the TMD functions. In the near future more SIDIS data will be collected by JLab at $12 \mathrm{GeV}$, while COMPASS will present results for the first ever polarised Drell-Yan process in $\pi^{-} p$, to check the change of sign of the Sivers function. COMPASS will also provide new measurements of azimuthal asymmetries in SIDIS on a unpolarised liquid hydrogen target.

In an even more distant future, many projects have been proposed. The PAX experiment at FAIR aims to investigate Drell-Yan reactions in polarised antiproton-polarised proton scattering 
(very clean way to address the transversity functions, but very challenging). Fixed target DrellYan experiments scattering polarised protons on polarised protons are being planned at JPARC, in Japan, and at NICA, in Dubna. It is clear, however, that the future of this field strongly depends on the realization of the long time dreamed polarised electron-polarised proton collider. The US project is on his track as a joint BNL-JLab collaboration and has been approved as one of the 'Long range plans' of the NSAC.

\section{References}

[1] HERMES Collaboration, A. Airapetian et al., Phys. Rev. Lett. 94 (2005) 012002, hep-ex/0408013.

[2] HERMES Collaboration, A. Airapetian et al., Phys. Rev. Lett. 103 (2009) 152002, 0906.3918.

[3] COMPASS Collaboration, M.G. Alekseev et al., Phys. Lett. B692 (2010) 240, 1005.5609.

[4] COMPASS Collaboration, C. Adolph et al., Phys.Lett. B717 (2012) 376, 1205.5121.

[5] Belle Collaboration, K. Abe et al., Phys. Rev. Lett. 96 (2006) 232002, hep-ex/0507063.

[6] I. Garzia et al., Nuovo Cimento 33C (2010) 269.

[7] M. Diehl and S. Sapeta, Eur. Phys. J. C41 (2005) 515, hep-ph/0503023.

[8] A. Bacchetta et al., JHEP 02 (2007) 093, hep-ph/0611265.

[9] X. Ji, J. Ma and F. Yuan, Phys. Rev. D71 (2005) 034005, hep-ph/0404183.

[10] X. Ji et al., Phys. Rev. D73 (2006) 094017, hep-ph/0604023.

[11] H. Avakian, A. Bressan and M. Contalbrigo, Eur. Phys. J. A52 (2016) 150, [Erratum: Eur. Phys. J.A52,no.6,165(2016)].

[12] C.A. Aidala et al., Rev.Mod.Phys. 85 (2013) 655, 1209.2803.

[13] V. Barone, F. Bradamante and A. Martin, Prog.Part.Nucl.Phys. 65 (2010) 267, 1011.0909.

[14] J.C. Collins, Nucl. Phys. B396 (1993) 161, hep-ph/9208213.

[15] COMPASS Collaboration, V.Y. Alexakhin et al., Phys. Rev. Lett. 94 (2005) 202002, hep-ex/0503002.

[16] COMPASS Collaboration, M. Alekseev et al., Phys. Lett. B673 (2009) 127, 0802.2160.

[17] COMPASS Collaboration, C. Adolph et al., Phys. Lett. B744 (2015) 250, 1408.4405.

[18] M. Anselmino et al., Phys.Rev. D75 (2007) 054032, hep-ph/0701006.

[19] M. Anselmino et al., Nucl. Phys. Proc. Suppl. 191 (2009) 98, 0812.4366.

[20] COMPASS, C. Adolph et al., Phys. Lett. B736 (2014) 124, 1401.7873.

[21] A. Kotzinian, Phys. Rev. D91 (2015) 054001, 1408.6674.

[22] COMPASS, C. Adolph et al., Phys. Lett. B753 (2016) 406, 1507.07593.

[23] COMPASS Collaboration, C. Adolph et al., Phys.Lett. B717 (2012) 383, 1205.5122.

[24] M. Anselmino, M. Boglione and S. Melis, Phys.Rev. D86 (2012) 014028, 1204.1239.

[25] P. Sun and F. Yuan, Phys.Rev. D88 (2013) 114012, 1308.5003.

[26] M.G. Echevarria et al., Phys.Rev. D89 (2014) 074013, 1401.5078. 
[27] COMPASS, A. Szabelski, EPJ Web Conf. 85 (2015) 02006.

[28] COMPASS, K. Kurek and A. Szabelski, J. Phys. Conf. Ser. 678 (2016) 012055.

[29] COMPASS Collaboration, C. Adolph et al., Phys. Lett. B718 (2013) 922, 1202.4064.

[30] COMPASS Collaboration, C. Adolph et al., Phys. Rev. D87 (2013) 052018, 1211.6849.

[31] S.J. Brodsky and S. Gardner, Phys. Lett. B643 (2006) 22, hep-ph/0608219.

[32] M. Anselmino et al., Phys.Rev. D74 (2006) 094011, hep-ph/0608211. 